\title{
Ultrasonic Power and Data Transfer through Multiple Curved Layers Applied to Pipe Instrumentation
}

\author{
Victor L. Takahashi ${ }^{1, *(0)}$, Alan C. Kubrusly ${ }^{2} \oplus$, Arthur M. B. Braga ${ }^{1}{ }^{\circledR}$, Sully M. M. Quintero ${ }^{1}$, \\ Sávio W. O. Figueiredo ${ }^{1}$ and Ana B. Domingues ${ }^{3}$ \\ 1 Department of Mechanical Engineering, Pontifical Catholic University of Rio de Janeiro, \\ Rio de Janeiro 22451-900, Brazil; abraga@puc-rio.br (A.M.B.B.); sully@puc-rio.br (S.M.M.Q.); \\ savio@puc-rio.br (S.W.O.F.) \\ 2 Center for Telecommunication Studies, Pontifical Catholic University of Rio de Janeiro, \\ Rio de Janeiro 22451-900, Brazil; alan@cpti.cetuc.puc-rio.br \\ 3 Shell Technology Department, Shell Brasil Ltd.a, Rio de Janeiro 20031-170, Brazil; A.Domingues@shell.com \\ * Correspondence: takahashi@puc-rio.br
}

Received: 23 August 2019; Accepted: 18 September 2019; Published: 20 September 2019

check for updates

\begin{abstract}
Ultrasonic power and data transfer through multilayered curved walls was investigated using numerical and experimental analysis. The acoustic channel used in this paper was formed by two concentric pipes filled with water, aiming for applications that involve powering and monitoring sensors installed behind the pipe walls. The analysis was carried out in the frequency and time domains using numerical and experimental models. Power and data were effectively simultaneously transferred through the channel. A remote temperature and pressure sensor was powered and interrogated throughout all the layers, and the power insertion loss was $10.72 \mathrm{~dB}$ with a data transmission rate of $1200 \mathrm{bps}$ using an amplitude modulated scheme with Manchester coding. The efficiency of the channel was evaluated through an experimental analysis of the bit error rate (BER) with different values of signal-to-noise ratio (SNR), showing a decrease in the number of errors compared with detection without Manchester coding.
\end{abstract}

Keywords: power and data transmission; ultrasonic communication; multiple layers acoustic transmission; passive sensor communication

\section{Introduction}

Ultrasonic waves can be used for wireless data communication and power transfer. Typical examples of well-developed practices include underwater acoustic communications [1-3], and biomedical applications where ultrasound is used to power and communicate with implantable devices [4-6]. The field of power and data transfer through metal layers using ultrasonic waves is, however, less well developed when compared to the aforementioned fields. Wireless transmission of power and data through metallic layers is relevant in applications where metallic walls cannot be penetrated, such as submarines [7] and pressure vessels [8]. Over the past 20 years, the use of an acoustic-electric channel on a metal barrier has been investigated [9], revealing a higher efficiency of power and data transfer when compared to electromagnetic-based solutions, either based on inductive [10-12] or capacitive [13] couplings. Several setups that rely on different types of transducers [14], channel configurations [8], and data modulations [10,15-17] have been investigated.

The most common setup consists of a pair of ceramic piezoelectric transducers encompassing a metal layer through which communication is intended. Adhesive epoxy is usually used to bond the transducers on the metal surfaces. The power and data communication through an acoustic channel typically contains three major components, namely the outside block, the inside block and 
the acoustic channel. One possible topology is the so-called reflected-power [18]. In this setting, information is sent from the inside block to the outside block, whilst energy is sent from the outside to the inside block. Communication is possible by varying the impedance of the piezoelectric transducer located at the inside block, which consequently changes the amplitude of the reflected wave $[15,17,18]$, and modulates the continuous wave signal sent from the outside block. Typical values of data rate and power transfer for this configuration are around hundreds of bps and hundreds of $\mathrm{mW}[9,15,18]$.

Usually the medium through which ultrasonic communication takes place consists only of a single metallic layer and bonding layers necessary to fix the transducers [9]. It is naturally expected that, if the medium is composed of several layers, then the communication efficiency would be reduced due to the impedance difference along the wave propagating path, which naturally introduces mismatches, and consequently, less power may be expected to cross the channel. There are, however, few published works on ultrasonic communication and power transfer across multilayered channels. Chakraborty et al. [19] analyzed the transmission of power and data in the case of multiple layers of different materials, in a steel-water-steel configuration. Two channels were characterized, the first with a steel thickness of $15.97 \mathrm{~mm}$ and a water layer of $88.3 \mathrm{~mm}$, and the second with a steel thickness of $10.92 \mathrm{~mm}$ and a water layer of $55.62 \mathrm{~mm}$. They experimentally showed a power transfer efficiency of $30 \%$ and a practical achievable data rate around $4 \mathrm{Mbps}$ using orthogonal frequency-division multiplexing (OFDM) modulation.

Another characteristic that directly impacts the efficiency of the ultrasonic wave transmission is the eventual curvature of a layer; a circular pipe or a metal curved shell could change the power and data transfer $[17,20]$. Analysis and characterization of ultrasonic channels that have curved metal walls is gaining attention. Chase [17] demonstrated the feasibility to transmit power and data through a curved metal wall made of aluminum. A pipe with an outer diameter of $76 \mathrm{~mm}$ and thickness of $3.2 \mathrm{~mm}$ was experimentally evaluated by attaching piezoelectric transducers on the pipe surface with a transition piece that matched the curvature of the tube.

When the electric impedance of the system was matched, a power transfer efficiency close to $30 \%$ and a data transfer rate of $10 \mathrm{kbps}$ were achieved. Ding Xin et al. [20] adopted a similar setup, but used a wider and thicker steel pipe with a $85 \mathrm{~mm}$ outer diameter and $10 \mathrm{~mm}$ thickness for power transfer only. The latter achieved $28 \mathrm{~dB}$ of insertion loss; considerably lower than Chase's [17] work, which reached $5.7 \mathrm{~dB}$.

A multilayered curved channel can be of interest in applications that concern transmission through concentric pipes. An example of such a scenario is found in the oil and gas industry, and, more specifically, in wellbore monitoring. This case consists of an inner and an outer pipe with liquid between them. The former is known as production tubing and the latter as casing pipe. One is usually interested in monitoring the structural health of regions beyond the casing layers. This can be achieved by placing sensors on the exterior of the casing pipe. Nevertheless, access is only possible in the interior of the tubing. Therefore, the issue is how to power supply the sensors and to get their data. A possible solution is to transfer power and to interrogate the sensor utilizing ultrasonic waves generated in the tubing. This scenario is, however, composed of a curved multilayer structure, being thus more complex than the aforementioned usual applications of ultrasonic through-wall communication. This paper investigates the potential of using a multi-layered structure composed of a water layer and a curved steel barrier aiming for a future wellbore monitoring system. Our experimental setup consisted of an arrangement of a pair of piezoelectric ultrasonic transducers enclosing multiple layers formed by two concentric pipes separated by water. In the inner pipe, a cover made of steel protects the piezoelectric transducer, adding, however, one more acoustic layer to the arrangement. The piezoelectric transducers have a nominal resonance frequency of $1 \mathrm{MHz}$, and the communication between then uses the impedance variation in order to amplitude modulate the carrier signal [17]. A complementary investigation was carried out by simulating the electro-acoustic channel using a one-dimensional multilayer electro-mechanical model designed in PSpice ${ }^{\circledR}[18]$. 
The remainder of the paper is organized as follows: Section 2 reviews power and data acoustic channel (PDAC) systems and the power and used data communication method. Section 3 describes the numerical and experimental acoustic channel analysis. Section 4 describes the system implementation and shows the experimental and numerical results. Section 5 concludes the paper.

\section{Power and Data Acoustic Channel System Overview}

The acoustic channel is composed of three major components, namely the outside block, inside block and acoustic channel, as Figure 1 illustrates. The outside block is composed of a signal generator and a radio frequency (RF) amplifier, both are responsible for sending a carrier signal to an ultrasonic transducer (made of lead zirconate titanate (PZT) ceramic), named the outside transducer, and a circuit to demodulate the received signal, based on an envelope detector. The inside block is composed of a circuit that rectifies and stores the received energy, a piezoelectric transducer (named the inside transducer), and a circuit to modulate the signal. The acoustic channel block is the medium connecting both sides by means of an ultrasonic wave propagating across it. Unlike the conventional application [14,18], in this paper the propagating medium is not composed of a single metallic flat layer. Instead, it presents a multilayer curved structure formed by two concentric tubes separated by water.

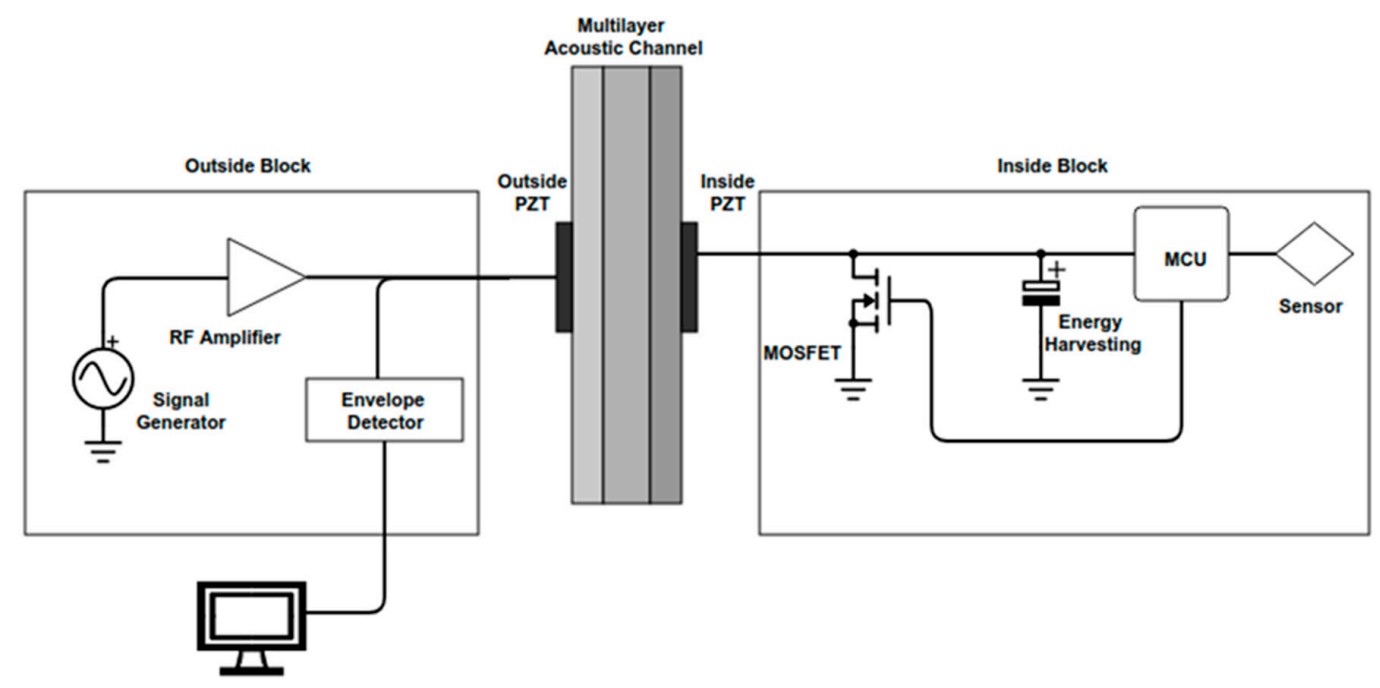

Figure 1. Power and data acoustic channel (PDAC) overall system diagram.

\section{Power and Data Communication Method}

The sensors and the complementary electronics that are located on the inside block are completely powered from the outside block through ultrasonic waves that reach the inside block. These acoustic waves are generated in the transmitting transducer, outside the PZT (shown in Figure 1), by a continuous wave $(\mathrm{CW})$ voltage that is applied at the outside the PZT's terminals (the signal generation and RF amplifier in Figure 1). The waves then propagate and impinge the face of the receiving transducer, inside the PZT (shown in Figure 1), in the form of vibration. Some energy is attenuated in the acoustic channel because of material losses $[18,21]$ and reflection due to an acoustic impedance mismatch between the layers across the channel; however, sufficient power reaches the inside block to supply the electronic components. The transducer in the inside block then converts the vibration to voltage. The electric energy is stored in the inside block in order to power all the necessary electronics and sensors.

The data communication principle is based on the impedance variation [15-18]. The digital information from the sensors is read by a microcontroller unit (MCU), which encodes it in two logic levels, and sends it to one of its pins. This pin is connected to the metal oxide semiconductor field effect transistor (MOSFET) transistor that short-circuits the terminals of the inside piezoelectric transducer to ground, as shown in Figure 1. By switching the transducer terminals between open and shorted, 
the acoustic impedance of the channel changes because the electrical terminal of the PZT has its boundary condition altered. Consequently, the equivalent electric impedance seen in the terminals of the outside PZT also, slightly, changes, which consequently varies the CW signal amplitude in the outside PZT's terminal. Therefore, an amplitude modulation scheme is achieved, and it can be demodulated by the outside block, using a simple envelope detector followed by a digital signal processor in order to interpret the sensors' data. Therefore, power is sent from the outside block to the inside block and data is sent from the inside block to the outside block using a single channel and carrier wave.

\section{Acoustic Channel Analysis}

\subsection{Numerical Model}

The electro-mechanical model used in this paper follows the approach developed by Roa-Prada et al. [20], which was implemented on an electrical circuit simulator. The whole acoustic channel, composed of piezoelectric transducers and intermediate layers, can be simulated by considering the electro-mechanical analogy between voltage and current to pressure and velocity, respectively. The 0piezoelectric transducer component is modeled as a three-port device, being one electrical and two mechanical (shown in Figure 2). The electric signal is applied or sensed in the electrical port (EP), whereas the two mechanical ports (MP), represent the front and back faces of the transducer, which vibrate when ultrasonic waves are generated or impinge upon them. One of the mechanical ports is terminated by a resistor, simulating the back face of the transducer in air. The intermediate layers are implemented as a purely two-port mechanical device.

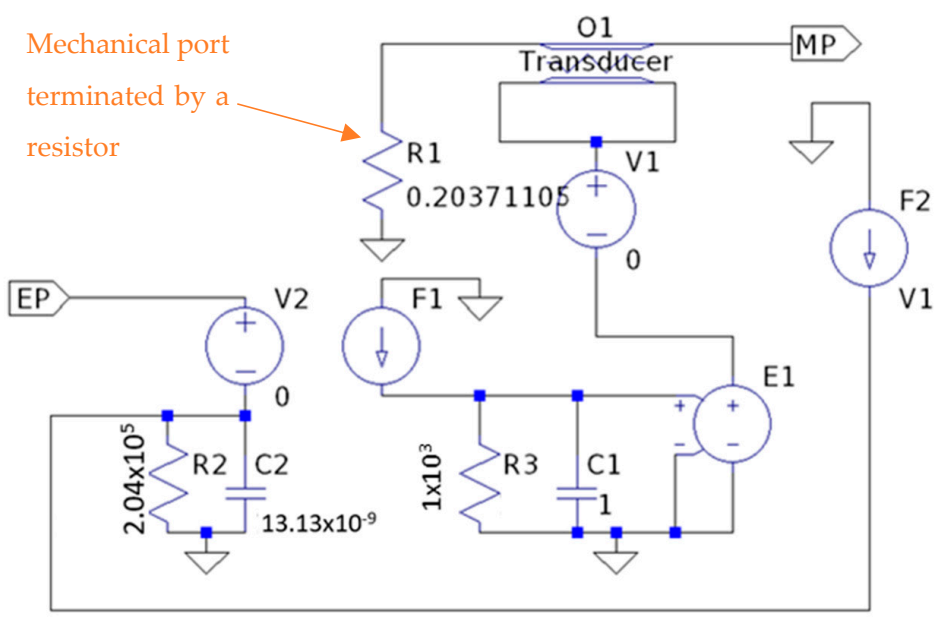

Figure 2. Equivalent electrical model of a piezoelectric ceramic transducer.

Figure 3 shows the diagram of the model designed in LTspice ${ }^{\circledR}$. As one can notice, in the acoustic channel block, the intermediate layers are modeled as transmission lines. The power analysis is performed by means of S-parameters, which are typical network parameters that relate the amount of power reflected or transmitted in each pair of ports [22]. In the present case, the S21 parameter of the whole channel indicates how much power a load in the inside block absorbs when a certain amount of power is injected in the outside block. Moreover, this circuit model approach can provide transient analysis such as instant voltage, current and power, which are useful to analyze the data communication system. Therefore, this model helps with understanding some behaviors of the electro-acoustic channel, such as power transfer and data communication, by evaluating the electrical signals on the electrical ports named EP2 and EP1. The material properties can be easily modified by changing the model parameters. An important advantage of the model is that multiple layers can be added or removed by simply connecting/disconnecting the new transmission lines. 


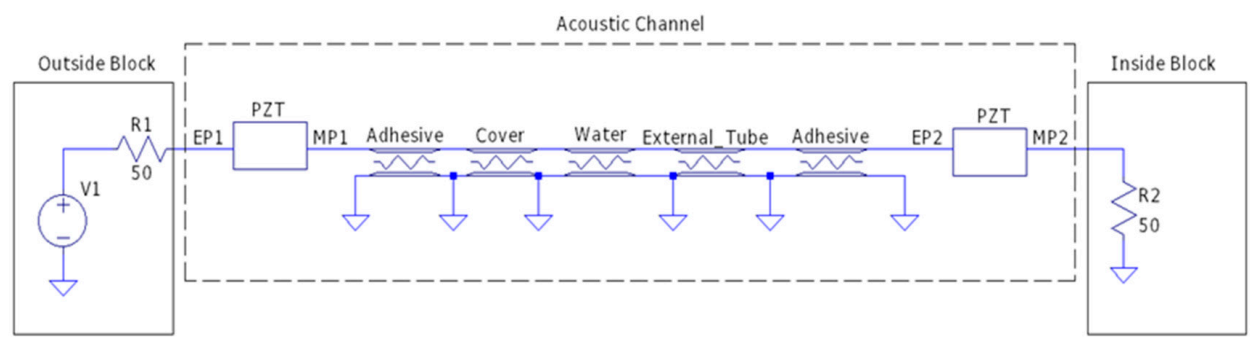

Figure 3. Ultrasonic channel formed by the intermediate layers and piezoelectric transducers. MP denotes mechanical port, whereas EP denotes electrical port. The element labeled PZT is the equivalent model of a piezoelectric transducer.

\subsection{Experimental Setup}

An experimental setup was created in order to investigate power and data transfer across multiple curved layers (water and steel). The setup has two concentric pipes with water in between them with dimensions shown in Figure 4a,b, respectively. The geometric parameters such as diameters and thickness of the pipes are based on the actual dimensions used in wellbore systems. For a proper operation, the piezoelectric transducers must be aligned to reduce the insertion loss of the acoustic channel $[17,18]$; this means that the energy transfer to the inside block is dramatically dependent on the alignment of both transducers. According to this, and knowing that the control of alignment between the transducers is difficult for a wellbore application, a higher area of the piezoelectric transducer was used to reduce the loss of power due to misalignment. The dimensions of the rectangular-shaped piezoelectric transducers were $70 \mathrm{~mm} \times 25 \mathrm{~mm} \times 2 \mathrm{~mm}$, and are also shown in the inset plot of Figure $4 \mathrm{a}$. Furthermore, generally the acoustic channel has a high selectivity in frequency [16-18], meaning that the system must operate in an optimum frequency that provides sufficient power to the inside block and an adequate voltage variation to allow a reliable communication. As it can be seen in Figure $4 b$, the experimental setup comprised a flat stainless steel case that covers the internal transducer, keeping its electrodes from being in contact with the water and also leaving the piezoelectric back face in contact with air. This case is $7.5 \mathrm{~mm}$ thick and is attached on the internal pipe. The water layer and the external pipe section are $20.5 \mathrm{~mm}$ and $20 \mathrm{~mm}$ thick, respectively. The external and internal pipes are made of stainless steel. The physical properties of the different materials used in the experimental setup are shown in Table 1. It is worth noticing that, despite the whole structure being cylindrical in nature, the PZTs were bonded to flat surfaces in order to provide better coupling for generated and received vibrations, as shown in Figure 4. Consequently, in what concerns the ultrasonic propagating path, there is only one curved interface in the channel, namely the interface at the inner radius of the outer steel pipe and the water annulus, as can be seen by the outer dashed circle in Figure 4a.

Table 1. Material properties.

\begin{tabular}{|c|c|c|c|c|c|}
\hline \multicolumn{3}{|c|}{ Piezoceramic: PZT5 } & \multicolumn{3}{|c|}{ Fluid: Water $\left(20^{\circ} \mathrm{C}\right)$} \\
\hline Density & $\rho\left(\mathrm{kg} / \mathrm{m}^{3}\right)$ & 7733 & Density & $\rho\left(\mathrm{kg} / \mathrm{m}^{3}\right)$ & 1000 \\
\hline Sound speed & $v(\mathrm{~m} / \mathrm{s})$ & 4419 & Sound speed & $v(\mathrm{~m} / \mathrm{s})$ & 1484 \\
\hline Quality factor & $\mathrm{Q}_{\mathrm{m}}$ & 48 & Acoustic damping coefficient & $\alpha_{\mathrm{V}}(\mathrm{Np} / \mathrm{m})$ & 0 \\
\hline Electrical permittivity & $\varepsilon_{33} \mathrm{~s}_{/} \varepsilon_{0}$ & 1101 & Thickness & $t_{W}(m)$ & $20.5 \times 10^{-3}$ \\
\hline Piezoelectric coupling factor & $\kappa_{33}$ & 0.58 & Area & $A\left(m^{2}\right)$ & $175 \times 10^{-3}$ \\
\hline Piezoelectric coefficient & $\mathrm{C}_{33}\left(\mathrm{~N} / \mathrm{m}^{2}\right)$ & $15.1 \times 10^{10}$ & & & \\
\hline Area & $\mathrm{A}\left(\mathrm{m}^{2}\right)$ & $175 \times 10^{-3}$ & & & \\
\hline Thickness & $\mathrm{t}(\mathrm{m})$ & $2 \times 10^{-3}$ & & & \\
\hline \multicolumn{3}{|c|}{ Metal: Stainless Steel } & \multicolumn{3}{|c|}{ Adhesive: Epoxy } \\
\hline Density & $\rho\left(\mathrm{kg} / \mathrm{m}^{3}\right)$ & 7894 & Density & $\rho\left(\mathrm{kg} / \mathrm{m}^{3}\right)$ & 1400 \\
\hline Sound speed & $v(\mathrm{~m} / \mathrm{s})$ & 5619 & Sound speed & $v(\mathrm{~m} / \mathrm{s})$ & 2344 \\
\hline Acoustic damping coefficient & $\alpha_{\mathrm{v}}(\mathrm{Np} / \mathrm{m})$ & 4 & Acoustic damping coefficient & $\alpha_{\mathrm{v}}(\mathrm{Np} / \mathrm{m})$ & 172 \\
\hline Area & $A\left(m^{2}\right)$ & $175 \times 10^{-3}$ & Area & $\mathrm{A}\left(\mathrm{m}^{2}\right)$ & $175 \times 10^{-3}$ \\
\hline Outer pipe thickness & $t_{\mathrm{O}}(\mathrm{m})$ & $7.5 \times 10^{-3}$ & Thickness & $\mathrm{t}(\mathrm{m})$ & $150 \times 10^{-6}$ \\
\hline Steel cover thickness & $t_{e}(m)$ & $20 \times 10^{-3}$ & & & \\
\hline
\end{tabular}




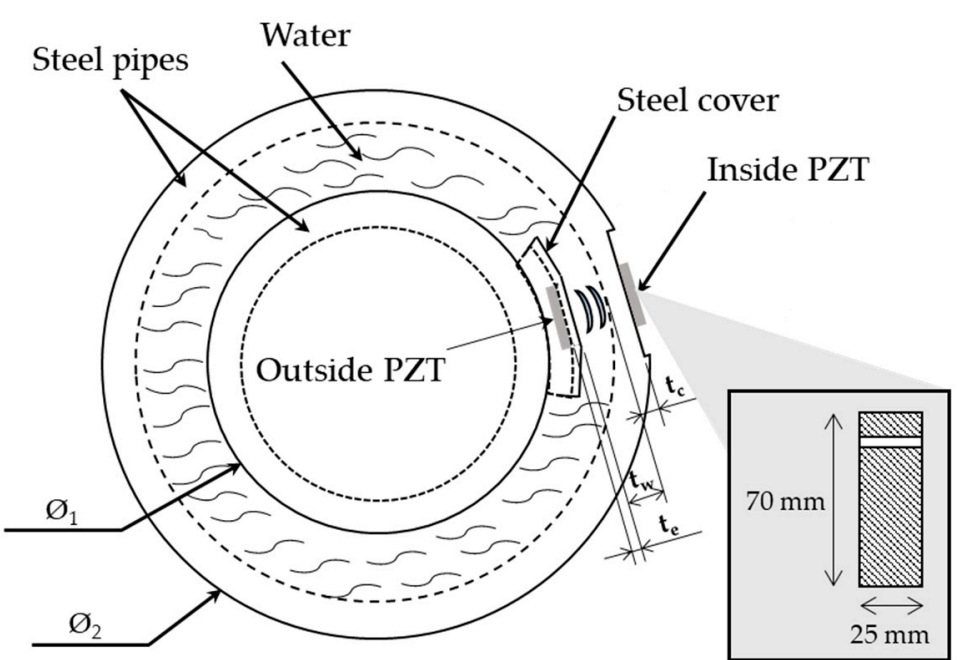

(a)

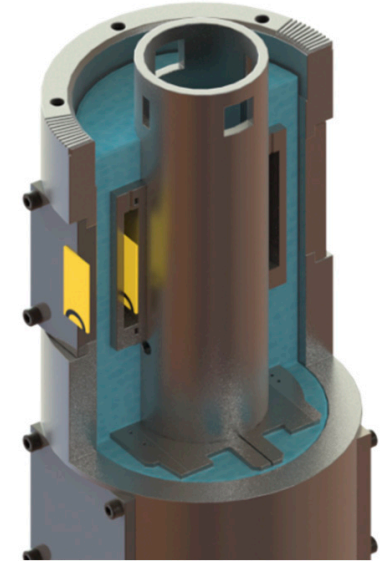

(b)

Figure 4. (a) Schematic diagram of the experiment: the outside transducer is bonded to the case, and the inside transducer is directly attached to the external pipe; (b) 3D isometric view of the experimental setup.

\subsection{Power and Data Communication Analysis}

In order to evaluate the power and data communication of the experimental setup, a vector network analyzer (VNA) was used. The S21 scattering parameter was measured and used to evaluate the power transfer. With this equipment, it is also possible to measure the input impedance of the system. Therefore, from the curves of impedance for the two cases, open and shorted, the input voltage variation $(V V)$ was calculated using Equation (1), through the difference of the input voltage when varying the input impedance

$$
V V=\frac{\left(\left|Z_{o}-Z_{c}\right|\right)}{\left(50+Z_{o}+Z_{c}\right)+\left(Z_{o} \times Z_{c}\right) / 50} \times V
$$

where $Z_{o}$ is the input impedance when the transducer is open-circuited, $Z_{c}$ is the input impedance when the transducer is short-circuited, and $V$ is the voltage applied to the transducer. Equation (1) is obtained by considering that the input voltage in each case is provided by a voltage divider circuit between a $50 \Omega$ nominal impedance and the input impedance. One can notice that the amplitude of the input voltage variation is proportional to the applied voltage $V$ and the impedance difference $\left(\left|Z_{o}-Z_{c}\right|\right)$. A $50 \Omega$ resistor, the output impedance of the VNA, is used in the calculation.

Figure 5a,b show the experimental and numerical results for the S21 scattering parameter and the input voltage variation, respectively. As it can be seen, there are several peaks in Figure 5 a due to the wave reverberation within the channel caused by reflections in between the layers and at the ends of the channel, thus creating several resonance frequencies in the working bandwidth, as commonly occurs in this type of ultrasonic communication system [9,21]. The simulated S21 curve of Figure 5a reasonably matches the more pronounced peaks of the experimental curves. Regarding the power transfer, the main maximums occur at $(1.118 \mathrm{MHz} ;-9.28 \mathrm{~dB})$ and $(1.119 \mathrm{MHz} ;-10.79 \mathrm{~dB})$ for the simulated and experimental curves, respectively, resulting in a difference of $1 \mathrm{kHz}$ in the frequency and $1.51 \mathrm{~dB}$ of the insertion loss. This amplitude difference may be due to differences between the actual and the tabled material properties used in the numerical model. Additionally, it is also possible to observe that the experimental curve has additional peaks with lower amplitudes between the multiple resonances of the channel that might be caused by other ultrasonic modes of propagation created inside the channel. It is also worth noticing that the simulated model, however, does not include 
the characteristics of a curved wall because it was developed to contemplate only one-dimension wave propagation $[23,24]$. With respect to the input voltage variation shown in Figure $5 b$, the main maxima occur at $(1.118 \mathrm{MHz} ; 0.43 \%)$ and $(1.119 \mathrm{MHz} ; 0.40 \%)$ for the simulated and experimental curves, respectively. In this case the difference between the input voltage variations is $0.03 \%$. It is straightforward to conclude that a good operating point is at $1.119 \mathrm{MHz}$ as it represents an operating point where both power transfer and data communication are augmented. It is worth noticing that if the propagating distance were larger, lower S21 values would be expected due to the higher attenuation along the channel. Low S21 is, obviously, an issue for power transfer because lower power arrives at the inside block. However, it is also complicating for data transmission even though communication relies on the difference of input voltage. The latter holds due to noise; if the absolute level is too low, the voltage variation may be undetectable. Thus, the propagation distance may be a limiting factor of this method if very long.

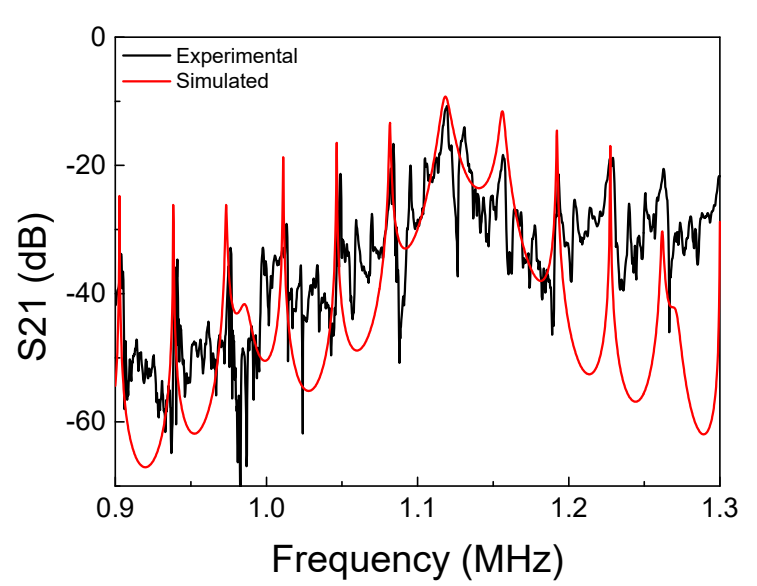

(a)

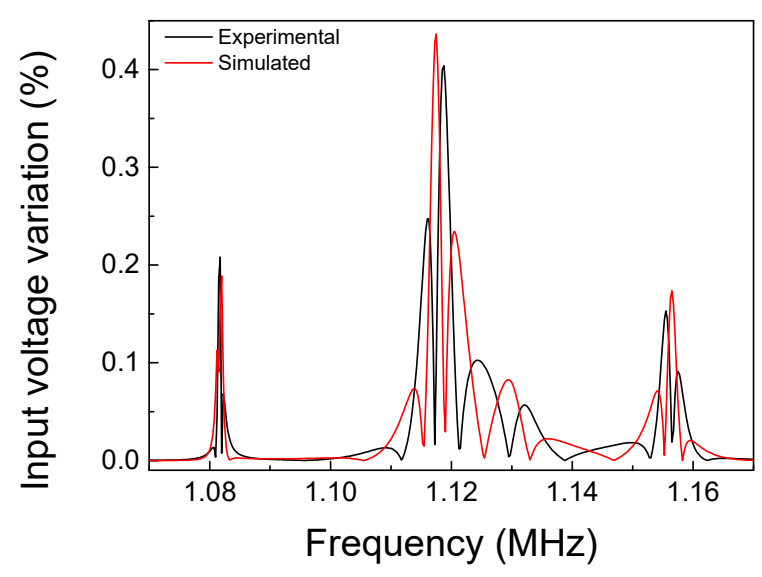

(b)

Figure 5. (a) Power transfer analysis and (b) input voltage variation of experimental and simulated results.

\section{System Implementation}

The electro-mechanical model presented in Section 3 is used to evaluate the wireless power transfer and data communication. An equivalent circuit simulates the electronic devices of the inside and outside blocks, and the multilayer acoustic channel, as illustrated in Figure 3. The outside block contemplates a signal source connected to an amplifier, and a demodulation circuit based on an envelope detector. The inside block, on the other hand, has a voltage doubler, a voltage regulator to supply the electronic components, and a MOSFET to perform the data communication.

\subsection{Full System Simulation}

The full system implementation designed on LTSpice, Figure 6, was implemented based on the experimental setup described in Section 3. The electrical signal applied to electrodes of the outside piezoelectric transducer was set to operate with an amplitude of $40 \mathrm{Vpp}$ at $1.119 \mathrm{MHz}$, where the maximum power transfer and voltage variation occur, according to Section 3.1. The whole system simulation is shown in Figure 6. In the outside block, in black, an envelope detector and a voltage comparator, in gray and brown, respectively, were designed. The output signal represents the data transmitted from the inside block. The isolated part of the system, the inside block, ensembles the components to conditionate the received power, the voltage doubler circuit, and the voltage regulator, in purple and dark green, respectively. The other components simulate the data transmission, here chosen as a digital pulser and n-channel MOSFET, in light green and orange, respectively. The digital pulser has a transmission rate of $4000 \mathrm{bps}$ and the output is directly connected to the MOSFET gate. Figure 7 shows three waveforms acquired from a full-system simulation, where the cyan one represents 
the voltage that is received by the inside transducer, the purple is the signal after the carrier signal passes through the envelope detector, and lastly, in black, the demodulated signal, which is the digital data when the enveloped signal passes through the voltage comparator. The generated voltage on the inside transducer has a maximum baseline of approximately $14 \mathrm{~V}$, and reaches around $1 \mathrm{~V}$ when the MOSFET is conducting. Due to the use of non-ideal elements implemented in the simulation, the minimum baseline voltage has a non-null voltage, as expected from the MOSFET on-resistance. The enveloped signal varies by approximately $1.5 \mathrm{~V}$ after passing through the envelope detector circuit, while the demodulated signal has a squared waveform of the same frequency and amplitude of the digital pulser.

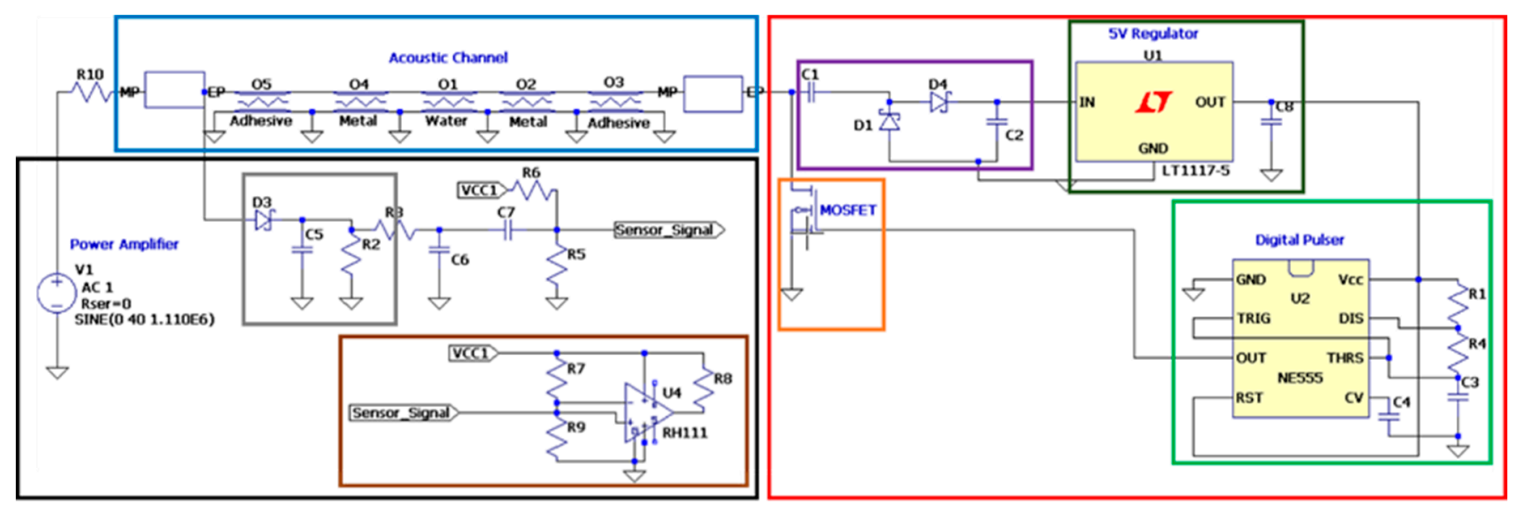

$\square$ Acoustic Channel $\square$ Outside Block $\square$ Inside Block $\square$ Envelope Detector $\square$ Voltage Comparator $\square$ Voltage Doubler $\square$ Voltage Regulator $\square$ Digital Pulser $\square$ MOSFET

Figure 6. Overall LTSpice schematic system.

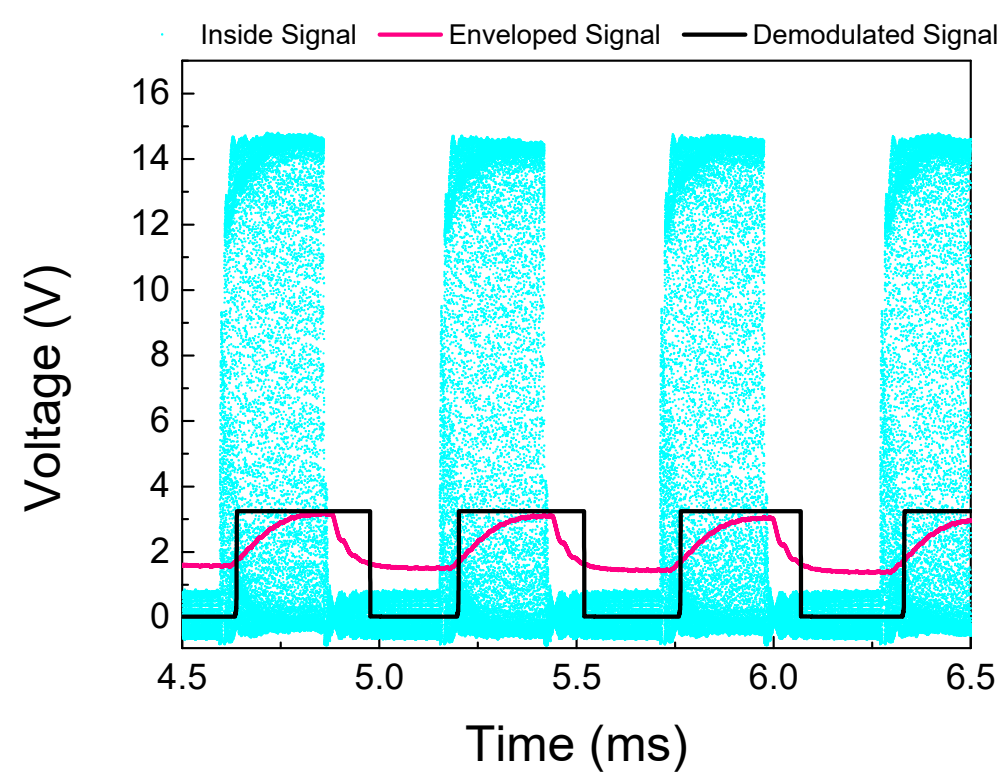

Figure 7. Results of the electrical model.

\subsection{Multilayer Curved Wall Full System Experiment}

Figure 8 shows the experimental setup. The inside and outside blocks, as well as the other components, are highlighted. In the inside block, a pressure and temperature sensor from Openfield replaces the digital pulser of the diagram in Figure 6 and a low-power microcontroller, MSP430F2619 from Texas Instruments ${ }^{\circledR}$, Thief River Falls, MN, USA, acquires the data of the sensor and generates asynchronous information based on Manchester coding [25]. The sensor, located in the inside block, is interrogated once per second, characterizing a low-power mode. The energy storage circuit guarantees the necessary power for the inside block when the transistor is in the on-state. The outside 
block has an envelope detector circuit and a digital signal processing code implemented inside the MCU to conclude the demodulation of the sensor data. The carrier frequency of the system is generated using a signal generator NI-VirtualBench model 8012, and amplified by a power amplifier before driving the outside piezoelectric transducer. After rebuilding the digital information of the sensor, the data is sent to a computer via a serial port.
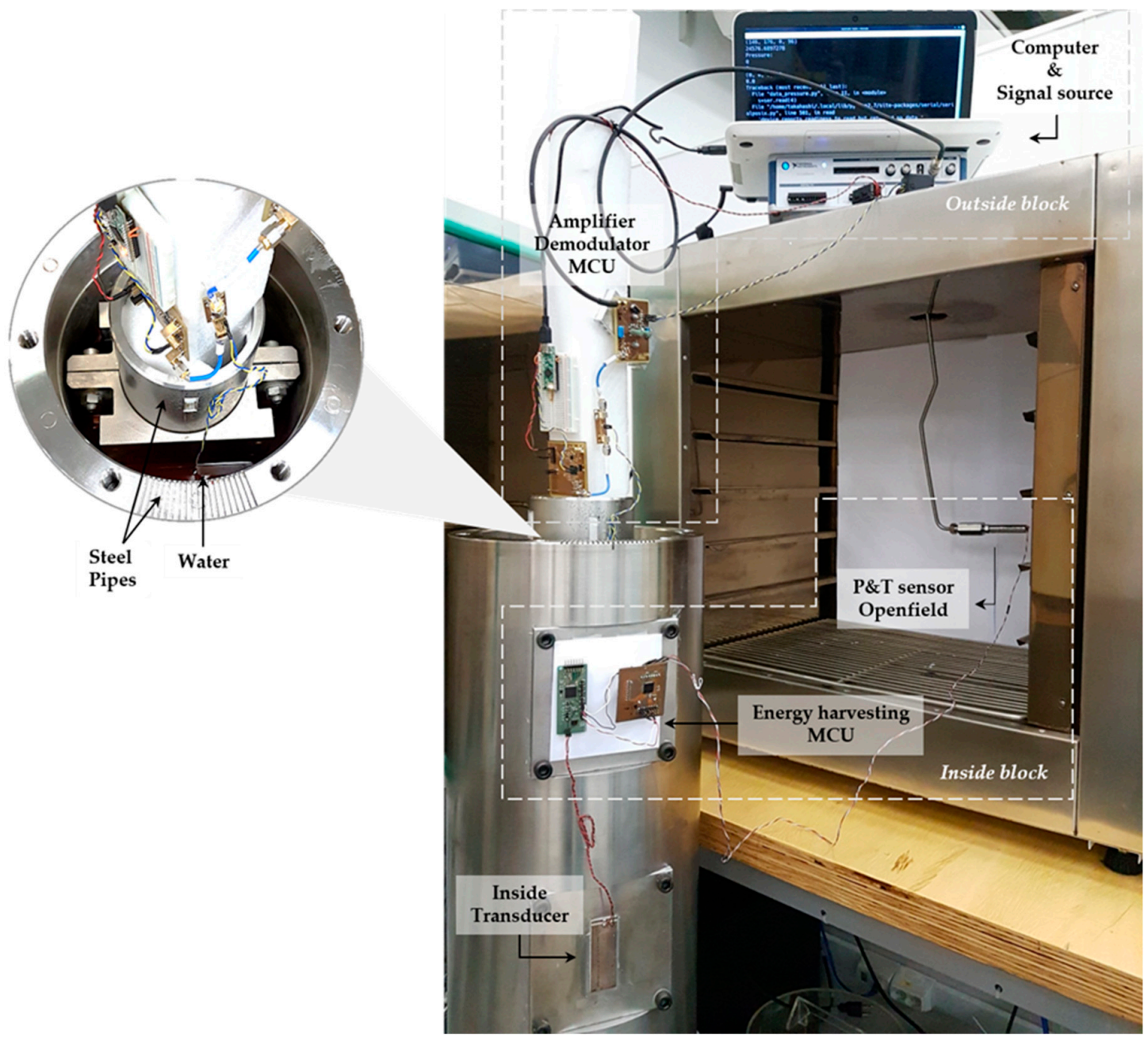

Figure 8. Experimental setup.

The voltage signals shown in Figure 9 were measured using a digital oscilloscope model MDO3024 from Tektronix ${ }^{\circledR}$. A typical voltage signal produced on the inside block by the inside transducer is presented in Figure 9b. Such as what occurred in the simulation analysis, the voltage has two levels depending on the state of conduction of the MOSFET. These two levels correspond to the sensor data rate of $1200 \mathrm{bit} / \mathrm{s}$. The high level baseline is approximately $10 \mathrm{~V}$; sufficient to power the electronic components even when the energy supply is almost null due to the MOSFET short-circuited state. The outside signal in purple in Figure $9 \mathrm{~b}$ is the voltage after the envelope detector circuit. This is the first stage of demodulation, and typical values of the difference in voltage amplitude range from $150 \mathrm{mV}$ to $500 \mathrm{mV}$, approximately, depending on the input voltage.

The transmission of the sensor data between the inside and the outside is implemented using amplitude shift keying (ASK) modulation [25], varying the impedance of the internal transducer. The data flows in only one direction, from the inside to the outside. However, the reverse direction would also be possible, working as a half-duplex, by varying the amplitude of the carrier in such a way to identify which side is transmuting, either inside or outside. 


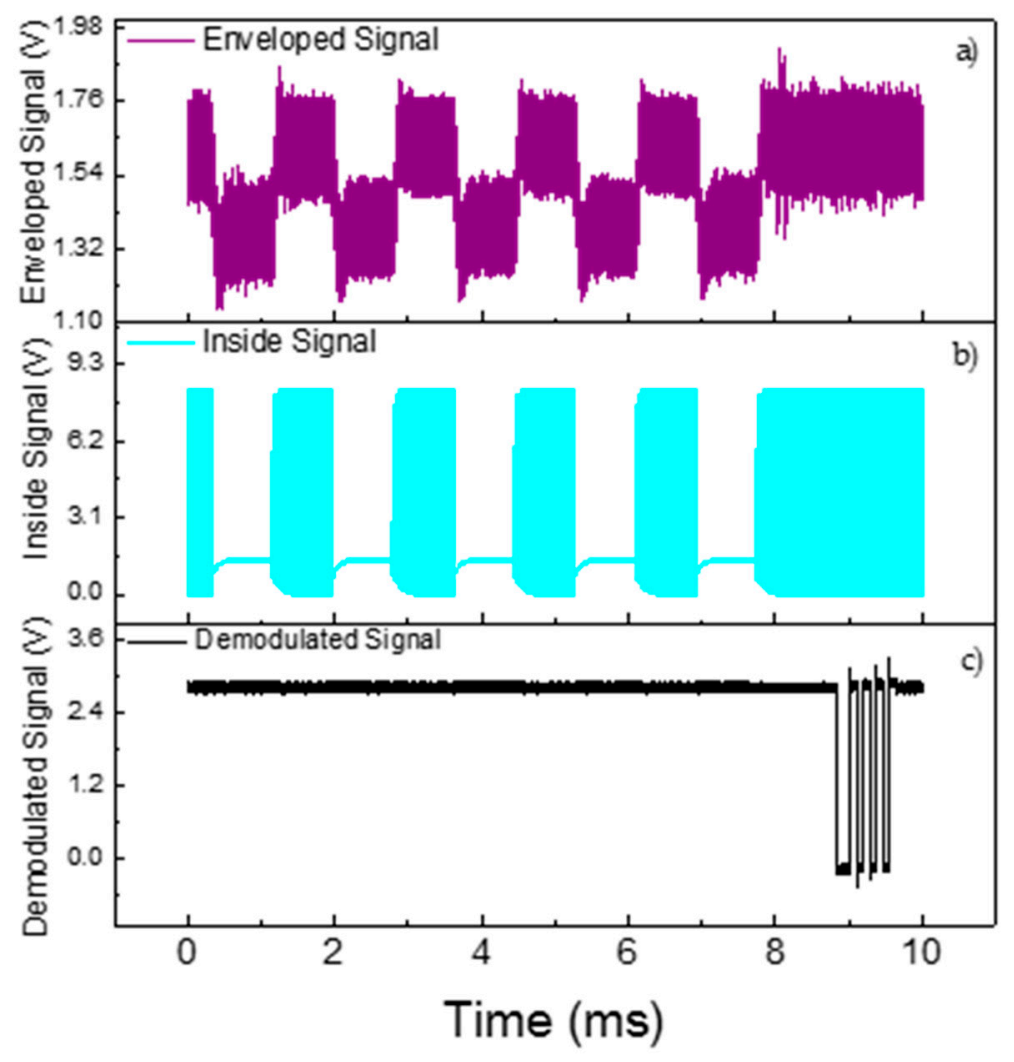

Figure 9. Oscilloscope signals showing (a) the enveloped signal after the envelope circuit detector in purple (500 $\mathrm{mV} / \mathrm{div}),(\mathbf{b})$ the inside voltage signal that reaches the inside transducers in cyan $(5 \mathrm{~V} / \mathrm{div})$ and (c) the demodulated signal processed inside a MCU and transmitted to an output pin (5 V/div).

In the reception, the demodulation is implemented using a non-coherent circuit, which means that the modulated signal passes through an envelope detector and a low-pass filter. In most common circuit demodulations, a comparator with a threshold level reconstructs the digital signal; however, in our system, the amplitude variation of the demodulated signal is often low, approximately $300 \mathrm{mV}$. Other characteristics of the system that affect detection capability, other than the low signal, include the dynamic DC level of the signal in the reception. In the idle mode, when no bits are being transmitted, the DC level is constant; however, when the inside block starts streaming the data, the direct current (DC) level decreases to balance the signal and therefore would require different threshold levels for a proper demodulation. The system is also affected by the amount of power that reaches the inside block, which is directly related to the power capacity to activate the MOSFET. These characteristics make it difficult to have a static threshold level on the comparator, increasing the error detection. The solution, therefore, was to use Manchester coding, which is less susceptible to noise and variations in DC level data. Thus, after passing through the envelope detector and low pass filter, the signal is inserted into the analog-digital converter (ADC) microcontroller to complete the second stage of demodulation using a digital processing unit. A sampling rate of $60 \mathrm{kS} / \mathrm{s}$ and a moving average of four samples of unwanted noise present after the envelope detector is effectively removed was used, making it possible to demodulate signals with poor signal-to-noise ratio. Furthermore, the use of Manchester coding favors the digital demodulation inside the MCU by using edge transition instead of level voltage to demodulate each bit. The black signal in Figure $9 \mathrm{c}$ is the reconstructed sensor data measured at the on-output pin of the microcontroller after the enveloped signal has been processed. The digital signal has a speed of $115 \mathrm{kbps}$ in the universal asynchrounous receiver/transmitter (UART) protocol, and it can be connected to any computer or data analyzer using UART-USB converters. 


\subsection{SNR and BER Analysis}

In this section an experimental procedure was performed to analyze the data transmission error as a function of the signal-to-noise ratio (SNR). The quantity of error in the reception was acquired by comparing the raw data before the transmission through the acoustic channel, and the received signal. Both raw and received data were bit compared one-by-one, which gives the bit error rate (BER), where the data rate was fixed at $64 \mathrm{bps}$. The SNR was measured by separating the energy of the signal and the energy of the noise. The signal, measured after the envelope detector circuit, contains the sum of the data signal and noise; however, as the raw data acquired on the transmitter mostly has the energy of the data signal, the noise energy is isolated through a fast Fourier transform (FFT) operation, removing the raw data frequency bins of the data signal. The missed frequency bins, from the noise signal, are therefore interpolated to have all of the frequency components. The amplitude levels of the signal data are summed, where the DC component is removed, and divided by the sum of the noise amplitudes, approximately estimating the SNR.

For a fixed SNR, the quantity of the bit error was acquired. The SNR was varied over different values of frequency instead of varying the input voltage of the power amplifier, and so maintaining almost the same noise level. One can notice that slightly changing the operating frequency does not interrupt the functionality of the system, but changes the input voltage variation as shown in Figure $5 \mathrm{~b}$. The frequency variation, therefore, increases or decreases the energy of the signal data in the reception.

Figure 10 shows the experimental and theoretical results of BER versus SNR. The theoretical curve, resulting from Equation (2), represents the probability of a bit error rate for a BASK modulation when the variation between the high level and the low level, bit 1 and 0 , respectively, is $18 \%$ [26]. This variation represents the best value in our experimental results, approximately at $13.5 \mathrm{~dB}$ with $10^{-6}$ of bit error rate. The experimental analysis also shows the results when the voltage variation between the high and low level is $5 \%$, at approximately $3 \mathrm{~dB}$ with $10^{-1}$ of bit error rate. Both signals are shown in Figure 11. In any case, it should be noted that, for our system, the error data rate is lower than the theoretical curve due to Manchester coding implementation.

$$
P_{e}(\operatorname{BASK}(18 \%))=\frac{1}{2} \operatorname{erfc}\left(\sqrt{\frac{3 \mathrm{SNR}}{80}}\right)
$$

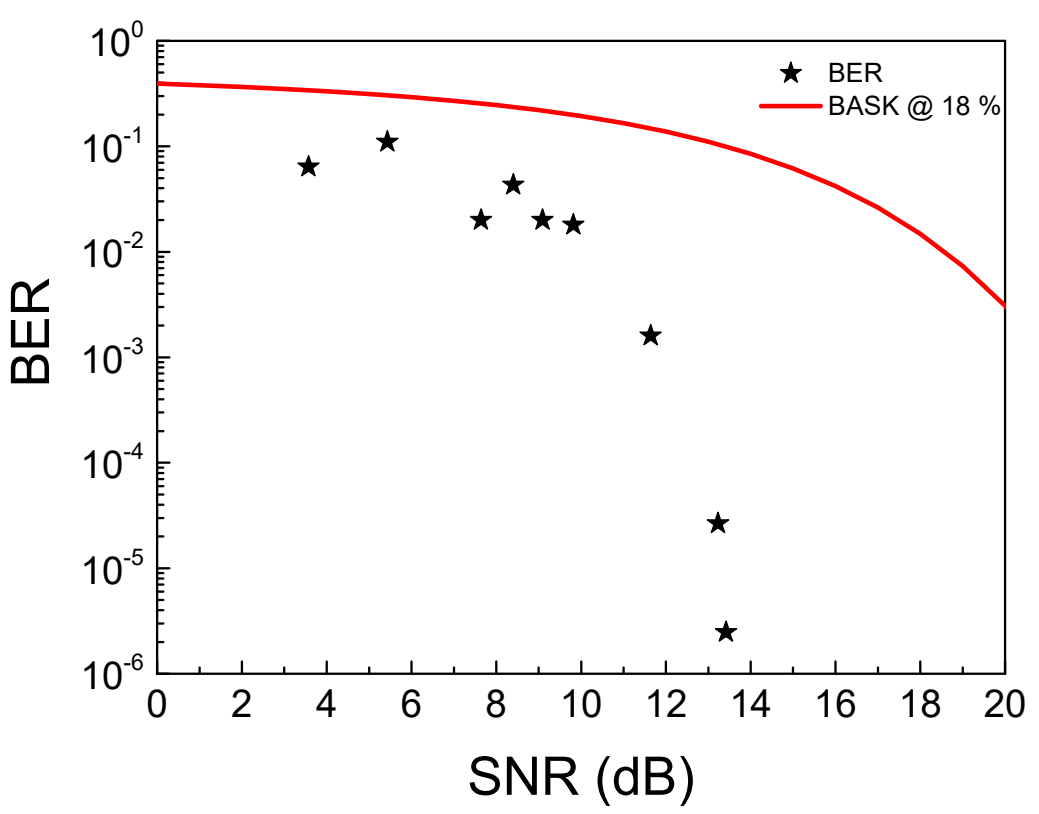

Figure 10. Experimental and theoretical BER curves. 


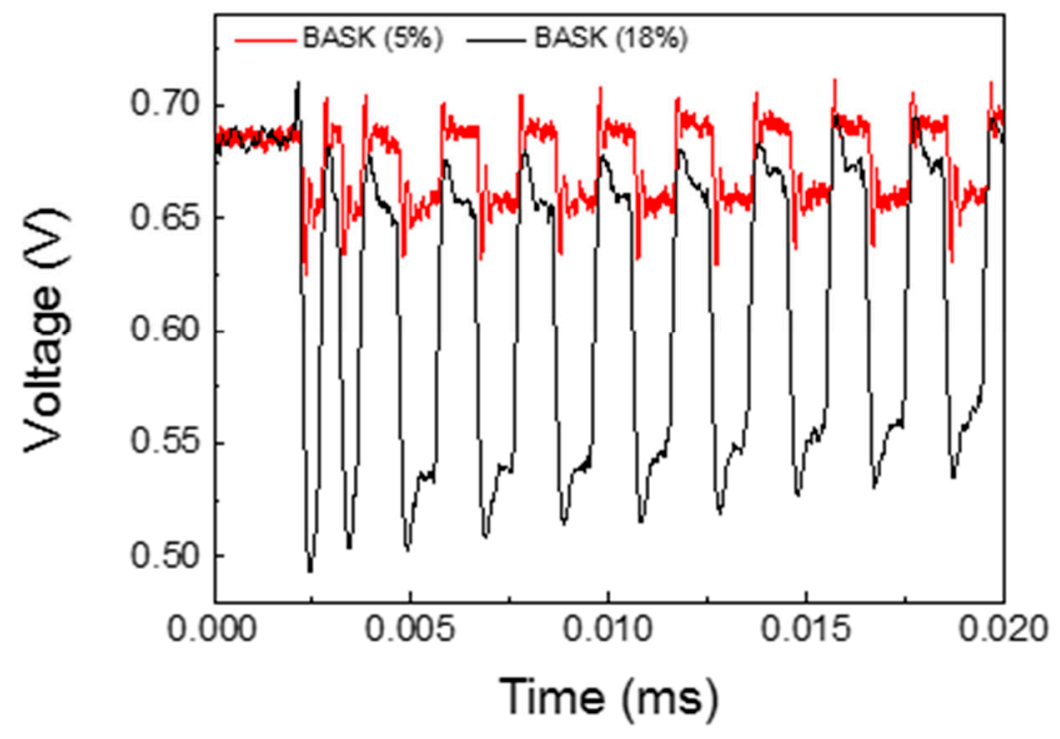

Figure 11. Received signal for BASK $5 \%$ and BASK $18 \%$.

\section{Conclusions}

Ultrasonic power and data were successfully transmitted through a multi-layered curved structure that was designed for wellbore monitoring. The pipe's geometry was based on the actual dimensions used in the field, as well as the direction of power and data. Power was sent from the inner pipe to the outer pipe, whereas data went on the reverse path, which is needed for wellbore monitoring systems. The experimental results showed the capability of the system to transfer the necessary energy to supply the electronics on the inside block, which, at full operating capability, has a power consumption of approximately $100 \mathrm{~mW}$, and also the capability to communicate with a digital sensor at a rate of 1200 bps using Manchester coding. The proposed solution also suggested that any digital sensor can be wirelessly interrogated through the acoustic channel. The implementation of Manchester coding and the MCU also allowed the system to operate in a noisy condition compared with the detection method of the analog voltage system. The system was able to demodulate the sensor information with extremely low values of the enveloped signal (approximately $150 \mathrm{mV}$ ) when using a digital low pass filter implemented on the MCU. The proposed method is, therefore, promising for field applications with wellbore monitoring systems.

In order to increase the comprehension of the acoustic channel behavior, future studies on how the misalignment between transducers and temperature affect the power and data transmission of the system need to be conducted. Furthermore, the use of techniques to increase the efficiency, such as electrical impedance matching and other types of modulation to increase the channel capability, need to be investigated.

Author Contributions: Conceptualization, V.L.T. and A.M.B.B.; Formal analysis, V.L.T. and A.M.B.B.; Funding acquisition, A.B.D.; Investigation, A.C.K., S.M.M.Q. and S.W.O.F.; Methodology, A.C.K.; Project administration, A.B.D.; Software, V.L.T.; Supervision, A.M.B.B.; Validation, S.M.M.Q. and S.W.O.F.; Writing—original draft, V.L.T., A.C.K., A.M.B.B., S.M.M.Q., S.W.O.F. and A.B.D.; Writing-review \& editing, V.L.T., A.C.K., A.M.B.B., S.M.M.Q., S.W.O.F. and A.B.D.

Funding: This research was funded by Shell Brazil through the project BG-40.

Acknowledgments: This research was carried out in association with the ongoing R\&D project registered as ANP 19062-9, "MEMS-Wellbore Monitoring Systems" (PUC-Rio/Shell Brasil/ANP)-Sistema de monitoramento para poços baseado em dispositivos microeletromecânicos (MEMS), sponsored by Shell Brasil under the ANP R\&D levy as "Compromisso de Investimentos com Pesquisa e Desenvolvimento".

Conflicts of Interest: The authors declare no conflict of interest. 


\section{References}

1. Santos, R.; Orozco, J.; Micheletto, M.; Ochoa, S.F.; Meseguer, R.; Millan, P.; Molina, C. Real-Time Communication Support for Underwater Acoustic Sensor Networks. Sensors 2017, 17, 1629. [CrossRef] [PubMed]

2. Cañete, F.J.; López-Fernández, J.; García-Corrales, C.; Sánchez, A.; Robles, E.; Rodrigo, F.J.; Paris, J.F. Measurement and Modeling of Narrowband Channels for Ultrasonic Underwater Communications. Sensors 2016, 16, 256. [CrossRef] [PubMed]

3. Pranitha, B.; Anjaneyulu, L. Review of research trends in underwater communications-A technical survey. In Proceedings of the 2016 International Conference on Communication and Signal Processing (ICCSP), Melmaruvathur, India, 6-8 April 2016; pp. 1443-1447.

4. Arra, S.; Leskinen, J.; Heikkila, J.; Vanhala, J. Ultrasonic Power and Data Link for Wireless Implantable Applications. In Proceedings of the 2nd International Symposium on Wireless Pervasive Computing, San Juan, Puerto Rico, 5-7 February 2007; pp. 567-571.

5. Ozeri, S.; Shmilovitz, D. Ultrasonic transcutaneous energy transfer for powering implanted devices. Ultrasonics 2010, 50, 556-566. [CrossRef] [PubMed]

6. Sanni, A.; Vilches, A.; Toumazou, C. Inductive and Ultrasonic Multi-Tier Interface for Low-Power, Deeply Implantable Medical Devices. IEEE Trans. Biomed. Circuits Syst. 2012, 6, 297-308. [CrossRef] [PubMed]

7. Lee, S.Q.; Youm, W.; Hwang, G.; Moon, K.S.; Ozturk, Y. Resonant ultrasonic wireless power transmission for bio-implants. In Proceedings of the SPIE Smart Structures and Materials + Nondestructive Evaluation and Health Monitoring, San Diego, CA, USA, 9-13 March 2014.

8. Lawry, T.J.; Wilt, K.R.; Scarton, H.A.; Saulnier, G.J. Analytical modeling of a sandwiched plate piezoelectric transformer-based acoustic-electric transmission channel. IEEE Trans. Ultrason. Ferroelectr. Freq. Control 2012, 59, 2476-2486. [CrossRef] [PubMed]

9. Murphy, T.L. Ultrasonic Digital Communication System for a Steel Wall Multipath Channel: Methods and Results; Rensselaer Polytechnic Institute: Niskayuna, NY, USA, 2006.

10. Yang, D.X.; Hu, Z.; Zhao, H.; Hu, H.F.; Sun, Y.Z.; Hou, B.J. Through-Metal-Wall Power Delivery and Data Transmission for Enclosed Sensors: A Review. Sensors 2015, 15, 31581-31605. [CrossRef] [PubMed]

11. Imoru, O.; Jassal, A.; Polinder, H.; Nieuwkoop, E.; Tsado, J.; Jimoh, A.A. An Inductive Power Transfer through metal object. In Proceedings of the 2013 1st International Future Energy Electronics Conference (IFEEC), Tainan, Taiwan, 3-6 November 2013; pp. 246-251.

12. Zangl, H.; Fuchs, A.; Bretterklieber, T.; Moser, M.; Holler, G. An Investigation on Wireless Communication and Power Supply through Metal Tank Walls. In Proceedings of the 2008 IEEE Instrumentation and Measurement Technology Conference, Victoria, BC, Canada, 12-15 May 2008; pp. 1452-1457.

13. Zangl, H.; Fuchs, A.; Bretterklieber, T.; Moser, M.J.; Holler, G. Wireless Communication and Power Supply Strategy for Sensor Applications within Closed Metal Walls. IEEE Trans. Instrum. Meas. 2010, 59, 1686-1692. [CrossRef]

14. Liu, C.; Hu, A.P.; Nair, N.K.C. Coupling study of a rotary Capacitive Power Transfer system. In Proceedings of the 2009 IEEE International Conference on Industrial Technology, Gippsland, Australia, 10-13 February 2009; pp. 1-6.

15. Hosman, T.; Yeary, M.; Antonio, J.K.; Hobbs, B. Multi-tone FSK for ultrasonic communication. In Proceedings of the 2010 IEEE Instrumentation \& Measurement Technology Conference Proceedings, Austin, TX, USA, 3-6 May 2010; pp. 1424-1429.

16. Lawry, T.J.; Wilt, K.R.; Roa-Prada, S.; Ashdown, J.D.; Saulnier, G.J.; Scarton, H.A.; Das, P.K.; Gavens, A.J. A high-temperature acoustic-electric system for power delivery and data communication through thick metallic barriers. In Proceedings of the SPIE Defense, Security, and Sensing, Orlando, FL, USA, 25-29 April 2011.

17. Prada, S.R. Modeling, Design and Temperature Characterization of an Ultrasonic through-Wall Communication System; Rensselaer Polytechnic Institute: Ann Arbor, MI, USA, 2010.

18. Chase, R. Microcontroller Based Handheld Acoustic Communication \& Power Delivery through Metallic Barriers; Rensselaer Polytechnic Institute: Niskayuna, NY, USA, 2013.

19. Chakraborty, S.; Wilt, K.R.; Saulnier, G.J.; Scarton, H.A.; Das, P.K. Estimating channel capacity and power transfer efficiency of a multi-layer acoustic-electric channel. In Proceedings of the SPIE Defense, Security, and Sensing, Baltimore, MD, USA, 29 April-3 May 2013. 
20. Roa-Prada, S.; Scarton, H.A.; Saulnier, G.J.; Shoudy, D.A.; Ashdown, J.D.; Das, P.K.; Gavens, A.J. An Ultrasonic Through-Wall Communication (UTWC) System Model. J. Vib. Acoust. 2013, 135, 011004. [CrossRef]

21. Lawry, T. A High Performance System for Wireless Transmission of Power and Data through Solid Metal Enclosures; Rensselaer Polytechnic Institute: Troy, NY, USA, 2011.

22. Pozar, D.M. Microwave Engineering, 3rd ed.; John Wiley \& Sons Inc.: Hoboken, NJ, USA, 2007.

23. Van Deventer, J.; Lofqvist, T.; Delsing, J. PSpice simulation of ultrasonic systems. IEEE Trans. Ultrason. Ferroelectr. Freq. Control 2000, 47, 1014-1024. [CrossRef] [PubMed]

24. Puttmer, A.; Hauptmann, P.; Lucklum, R.; Krause, O.; Henning, B. SPICE model for lossy piezoceramic transducers. IEEE Trans. Ultrason. Ferroelectr. Freq. Control 1997, 44, 60-66. [CrossRef] [PubMed]

25. Lathi, B.P.; Ding, Z. Modern Digital and Analog Communication Systems; Oxford University Press: New York, NY, USA, 2019.

26. Thompson, R.A.; Tipper, D.; Krishnamurthy, P.; Kabara, J. The Physical Layer of Communication Systems; Artech House: Norwood, MA, USA, 2006.

(C) 2019 by the authors. Licensee MDPI, Basel, Switzerland. This article is an open access article distributed under the terms and conditions of the Creative Commons Attribution (CC BY) license (http://creativecommons.org/licenses/by/4.0/). 